\title{
Formen von Subjektivierung und Unbestimmtheit im Umgang mit datengetriebenen Lerntechnologien - eine praxistheoretische Position
}

\author{
Heidrun Allert • Michael Asmussen (D) Christoph Richter
}

Online publiziert: 11. September 2017

(C) Der/die Autor(en) 2017. Dieser Artikel ist eine Open-Access-Publikation.

Zusammenfassung Der Beitrag zielt darauf auszuloten, wie sich sozio-technische Phänomene in pädagogischen Kontexten mit Ansätzen sozio-materieller Praktiken verstehen lassen. Technologien werden darin als epistemische Objekte und produktive Dinge gefasst, da sie ko-konstitutives Element in sozialen Praktiken sind und laufend neue Fragen generieren. Hierzu wird zunächst das Mensch-Technik-Verhältnis performativ gefasst. In einem zweiten Schritt wird die Rolle von Daten und Algorithmen bei der Konstituierung des Subjekts diskutiert. In einem dritten Schritt wird das Gesagte am Beispiel Learning Analytics diskutiert. Zum Schluss wird eine Conclusio dargelegt, in der die Forderung, Materialität als ko-konstitutives Element in Praktiken auch in pädagogischer Theorie zu fassen, skizziert wird.

Schlüsselwörter Soziale Praktiken · Subjektivierung · Digitalität · Daten · Algorithmen

\section{Forms of Subjectivization and Uncertainty in the Use of Data-Driven Educational Technologies - a practice-theoretical position}

Abstract This article aims to conceptualize socio-technical systems as social practices. Artefacts are framed as epistemic objects and productive things which are constitutively entangled with human actors in social practices. As technology does

\footnotetext{
Prof. Dr. H. Allert · M. Asmussen, M.A. $(\varangle) \cdot$ Dipl.-Psych. C. Richter Institut für Pädagogik, Abteilung Medienpädagogik/Bildungsinformatik, Christian-Albrechts-Universität zu Kiel, Olshausenstraße 75, 24098 Kiel, Deutschland E-Mail: asmussen@paedagogik.uni-kiel.de

Prof. Dr. H. Allert

E-Mail: allert@paedagogik.uni-kiel.de

Dipl.-Psych. C. Richter

E-Mail: richter@paedagogik.uni-kiel.de
} 
not determine use, practices are performative, unpredictably open and generate phenomena and questions which cannot be derived from prior analyses and theory. Based on this perspective, the role of data and algorithms in social practices is discussed using the example of Learning Analytics. Finally, the article sets forth the argument of materiality as a concept in educational theory, as it is a co-constitutive element in educational practices.

Keywords Algorithms $\cdot$ Data $\cdot$ Digitality $\cdot$ Social practices $\cdot$ Subjectivization

\section{Einleitung}

In fiktionalen Erzählungen wie I, Robot (Asimov 1950) werden Technologien als Begleiter des Menschen mit anthropomorphen Zügen dargestellt, von denen wir uns immer weniger unterscheiden können. Wir interagieren mit diesen künstlichen Akteuren; sie stehen uns in Auseinandersetzungen gegenüber oder begleiten uns Seite an Seite. Sie werden erzählerisch und visuell als von uns unabhängige und eigenständige Entitäten als „,der Andere“ dargestellt. Es scheinen sich das Natürliche und das Künstliche, Natur und Technik, Mensch und Ding, Subjekt und Objekt dichotom gegenüber zu stehen. ${ }^{1}$

Es ist wiederum praktisch, Menschen so anzusehen wie Maschinen, wenn man die Formen der Interaktion von Mensch und Maschine in Arbeits- und Lernprozessen gestaltet als informationsverarbeitende und -speichernde Wesen, die Entscheidungen rational treffen, Faktoren miteinander verrechnen und aus Daten einzelner Vorkommnisse abstrahieren. Vielleicht zeigen aber gerade unsere Interaktionen mit Maschinen, dass wir anders sind. Sie werfen stetig die Frage auf, WIE wir Mensch sind und was es heißt, Mensch zu sein.

Die Dichotomie von Subjekt und Objekt ist hinterfragbar, denn wir stehen den künstlichen Akteuren nicht gegenüber, sondern werden mit ihnen zu dem, was wir sind. Die Praktiken, in denen wir mit Technologien konstitutiv verwoben sind, bilden unser Menschsein und die Subjektivierungsmodi in der Gesellschaft kontinuierlich neu. Menschsein können wir somit nur dynamisch verstehen und nicht statisch betrachten.

Dieser Beitrag möchte in Erfahrung bringen, (a) wie sich sozio-technische Phänomene mit Ansätzen sozio-materieller Praktiken bzw. aus praxeologischer Sicht fassen lassen und (b) wie wir Technologien als Erkenntnisobjekte verstehen können, die fortlaufend Fragen über unser Menschsein, über soziale Prozesse und unser Zusammenleben aufwerfen, Fragen, mit denen wir bei ihrer Entwicklung nicht gerechnet haben. Als Erkenntnisobjekte sind Technologien ,produktive Dinge“ (Knuuttila 2005, S. 69) - nicht weil ihre Nutzung Erkenntnisse oder Antworten liefern würde, sondern weil sie offene und komplexe Fragen stellt, die uns überraschen, weil wir sie bei ihrer Entwicklung nicht erkannt oder intendiert haben (vgl. Richter et al.

\footnotetext{
1 Natur ist, wie sie ist, so das Argument. Natur wird in pluralistischen Gesellschaften, in denen Werte heterogen bleiben und die sich selbst stetig neue Optionen schaffen, von manchen als unwandelbare ethische Autorität angeführt (vgl. Nowotny und Testa 2009).
} 
2015). Technologien repräsentieren nicht bloß ein Konzept, sie realisieren nicht nur eine Idee, sondern sind epistemische Objekte (vgl. Knorr Cetina 2001), insofern ihre Nutzung Fragen aufwirft, die wir im Vorfeld nicht hätten absehen und verhandeln können. Wir haben diese Fragen nicht in das Objekt implementiert - dennoch entfalten sie sich im Gebrauch.

Der folgende Text befasst sich zunächst mit der Absicht, das Mensch-TechnikVerhältnis performativ zu denken und mit der Bedeutung von Daten und Algorithmen im Bezug auf die Konstituierung des Subjekts, um dann das Gesagte am Beispiel Learning Analytics zu diskutieren und schließlich zu einer Conclusio zu gelangen, in der die Forderung, Materialität als konstitutives Element in Praktiken auch in pädagogischer Theorie zu fassen, skizziert wird.

\section{Subjekt und Technologie}

Für den pädagogischen Bezug auf Bildung und Erziehung sowie die Transformationsprozesse im Bildungssystem kann die Betrachtung der Beziehung Mensch Technologie grundlegende Fragen aufwerfen bzw. Grundfragen der Pädagogik erneut stellen. Was heißt Menschsein? Oder weniger naturalistisch und eher im gesellschaftlichen und politischen Sinne gefragt: Was heißt Subjekt sein und welche Modi der Subjektivierung lassen sich beim Heranwachsen, politischen Handeln, Arbeiten und Lernen in von Technologien geprägten Gesellschaften beschreiben?

Anstatt eine technikdeterministische Sichtweise zu verfolgen, nehmen wir hier eine relationale und transaktionale Perspektive ein: Wenn wir uns mit Technologien auseinandersetzen, sind wir unweigerlich vor die Frage gestellt, was es heißt, Mensch zu sein und erzeugen überhaupt erst Perspektiven auf uns selbst:

Wolfgang Pauser (1995, S. 26) spekuliert, dass das (gerade von der Technologiekritik immer wieder angeführte) romantische Sehnsuchtsbild ganzheitlicher zwischenmenschlicher Nähe womöglich weniger ein Erfahrungswert aus der Face-to-Face-Situation ist, sondern erst durch kognitive und/oder medial überbrückte Distanz denkbar und spürbar wird (Döring 2003, S. 170).

Der Gebrauch von Technologien ermöglicht uns Erkenntnisse über uns selbst, während wir gleichzeitig unser Selbst im Umgang mit ihnen transformieren. Sie sind epistemische Objekte und Transformatoren zugleich.

In der persönlichen Identitätsgenese ermöglicht uns die Nutzung von Medien Selbst- und Weltauseinandersetzung. In politischen Zusammenhängen stellt uns der Umgang mit und die Erfindung von Technologien die Frage neu, was es heißt Staatsbürger zu sein und was politisches Handeln sei. Dies betrifft etwa aktuell das Wissen um unser Unwissen bezüglich massenhafter Überwachung und die Möglichkeiten anonym zu handeln (vgl. de Lagasnerie 2016).

Die sich entfaltenden Fragen zu antizipieren, ist prinzipiell unmöglich, bevor die Dinge in Praktiken eintreten, da sie als epistemische Objekte partiell sind. In Praktiken sind menschliche Akteure und Dinge relational miteinander verbunden. ,,Der Nutzer' ist [...] alles andere als ein für sich bestehendes, Welt aneignendes Subjekt, sondern er ist ein Hybrid, das aus konkreten Relationierungspraktiken menschlicher 
und dinglicher Akteure hervorgeht" (Jörissen 2015, S. 228). Die Transformation können wir weder gedanklich vorwegnehmen, noch können wir uns theoretisch orientieren, solange das relationale Gefüge unverändert bleibt. Artefakte und „Nutzer“ sind keine isolierten Akteure, sondern in subjektivierenden Relationierungen zu verorten. „Eine Reflexion, die nicht mich in Relation auf andere involvierte Akteure transformiert, [sic!] und die somit nicht neue Assoziationen entstehen lässt, war keine Reflexion“ (Jörissen 2015, S. 228). Wir können Technologien als Artefakte ansehen, die unsere sozialen Praktiken sowohl transformieren als auch gleichzeitig erkennbar bzw. hinterfragbar und untersuchbar machen. Mensch und Technologie stehen sich nicht als unabhängige Entitäten gegenüber.

De Lagasnerie (2016, S. 76) spricht mit Bezug auf Foucault vom Subjektivierungsmodus, von neuen Weisen der Subjektivierung, von Praxis, die uns , über unsere gewöhnlichen Weisen lehren, Politik zu betreiben, sie begrifflich zu fassen - ja noch einfacher, uns selbst und unsere Verstrickung in die Welt und unsere Beziehungen zu anderen zu denken“. Technologien, die Menschen erfunden haben, ermöglichen neue Formen politischen Handelns, z. B. anonymes politisches Handeln: „Und was zeigt sich in einem umfassenden Sinne in dem Wunsch nach Anonymität, der sich im Internet und auch jenseits oder außerhalb der Politik in Alltagshandlungen äuBert?" (de Lagasnerie 2016, S. 75). Dieser Modus der Selbstkonstitution stellt die Art und Weise in Frage, wie wir Demokratie, politisches Handeln sowie eine bestimmte Idee des öffentlichen Raums und des kollektiven Ausdrucks denken, da politisches Handeln nicht mehr gleichzeitig mit dem öffentlichen in-Erscheinungtreten des Subjekts verbunden ist. Die Technologie ermöglicht einen Subjektivierungsmodus und eine Praxis, die die Begriffe gesellschaftlicher Theorie in Frage stellt.

Entsprechend betonen Ansätze sozio-materieller Praktiken die konstitutive Verwobenheit menschlichen Handelns mit Technologien. In einer praxeologischen Perspektive erscheint das Subjekt nicht als vorgängige und vorauszusetzende Entität, sondern als emergentes Resultat seiner Verstrickung in praktischen Vollzügen (vgl. Hörning 2001). Reckwitz (2009) hält fest, dass Praktiken nicht auf einem Subjekt basieren, sondern Akteure in Praktiken co-konstitutiv aufeinander verwiesen sind und hervorgebracht werden. Subjektivierung ist somit immer an die jeweiligen sozialen Praktiken gebunden. Gleichzeitig sind Praktiken aber nach Alkemeyer und Buschmann (2016) eine zu leistende Hervorbringung, die sich durch Kreativität und gestaltende Improvisation der Teilnehmer einer jeweiligen Praktik auszeichnet. Subjekte werden also durch Praktiken erzeugt, bringen aber auch ihr Gewordensein-inPraktiken und damit verbundene Erwartungen und Möglichkeiten (die sich in neuen Praktiken wieder verändern können) mit ein, sodass auch die Praktiken relational transformiert werden.

Sowohl de Lagasneries kritische Gegenwartstheorie als auch Ansätze sozio-materieller Praktiken denken in Praxis, die Struktur erzeugt. Theorien sozialer Praktiken betrachten Strukturen als etwas, das sich emergent aus dem Prozess des Handelns ergibt. Durch die relationalen Beziehungen aller beteiligten menschlichen und nichtmenschlichen Akteure in Praktiken wird kontinuierlich Realität erzeugt, die sich kontingent entfaltet. Neben den hieraus resultierenden Dynamiken wird uns im Weiteren insbesondere das Moment der Unbestimmtheit interessieren, das zunehmend auch in 
der Interaktion mit Technologien entsteht. Ebenso wie wir unsere Subjekthaftigkeit in Praktiken produzieren, entstehen auch die Qualitäten der Technologie emergent in der Nutzung. Da sich die Qualitäten der Technologien niemals vollständig vorab bestimmen lassen, wir nicht im Vorhinein wissen, wer wir sein werden und was sich verändern wird, wenn wir an entsprechenden Praktiken teilhaben, sind wir gezwungen zu handeln und zu interagieren, um die hieraus resultierenden kollektiven Praktiken in Erfahrung zu bringen. Handeln und Erkennen sind somit untrennbar ineinander verwoben.

Die hier skizzierte Perspektive unterscheidet sich sowohl von technikdeterministischen als auch kulturalistischen bzw. sozialdeterministischen Ansätzen, indem sie der gewöhnlich gedachten Subjekt-Objekt-Dichotomie ein relationales Verständnis entgegenstellt. Während in technikdeterministischen Ansätzen Technik ,zu einer gesellschaftsbestimmenden Macht“ (Belliger und Krieger 2006, S. 20) erklärt wird, durch deren gezielte Gestaltung soziale und gesellschaftliche Prozesse evoziert und gesteuert werden können, postulieren sozialdeterministische (manchmal auch kulturalistische) Ansätze, dass letztlich immer „Menschen und Gesellschaften entscheiden, ob eine Technik realisiert und wie sie eingesetzt wird." (Belliger und Krieger 2006, S. 21). Beide Positionen unterstellen, wenn auch mit umgekehrten Vorzeichen, die ontologische Trennung von Subjekt und Objekt. Theorieansätze soziomaterieller Praktiken gehen hingegen von einem „constitutive entanglement“ von Subjekt und Technik aus (Orlikowski 2007, S. 1437), in dem die Qualitäten von Technologien wie auch die Formen der Subjektivierung erst im praktischen Vollzug erkennbar werden ${ }^{2}$.

\section{Daten, Algorithmen und die Konstituierung des Subjekts}

Aufbauend auf den vorangestellten Überlegungen zur Verwobenheit von Subjekt und Technologie diskutieren wir im Folgenden die Bedeutung von Daten und Algorithmen im Bezug auf die Konstituierung des Subjekts und skizzieren die sich hieraus ergebenden pädagogischen Fragestellungen. Von besonderem Interesse ist dabei für uns die den entsprechenden Praktiken inhärente Unbestimmtheit und die damit einhergehenden individuellen und kollektiven Handlungsspielräume.

Die Verarbeitung von Daten ist nicht allein Voraussetzung aller modernen Informations- und Kommunikationstechnologien, sondern zugleich Gegenstand tiefgreifender politischer und ökonomischer Auseinandersetzungen. Daten werden zunehmend als eine wertvolle Ressource betrachtet, die es systematisch zu erschließen und wirtschaftlich zu nutzen gelte (vgl. Balkan 2016). Vor dem Hintergrund ökonomischer Verwertungsinteressen werden dabei auch zunehmend Einschnitte zum Schutz bzw. Veränderungen im Umgang mit personenbezogenen Daten erörtert. So forderte etwa Bundeskanzlerin Angela Merkel auf dem CDU-Parteitag 2016 den Abschied von den Prinzipien der Datensparsamkeit und der Zweckbindung und proklamier-

\footnotetext{
2 Die Unbestimmtheit der Qualitäten einer Technologie in Bezug auf ihren praktischen Gebrauch impliziert jedoch keine Beliebigkeit der Nutzung. Vielmehr schränkt die Materialität eines Artefakts oder die Struktur des Digitalen die Möglichkeiten des Gebrauchs ein.
} 
te stattdessen Datenreichtum als Gebot der Stunde ${ }^{3}$. Die Bedeutung datenbasierter Dienste ist in ihrer Dimension neu und wirft grundlegende Fragen auf, nicht zuletzt für die Pädagogik. Wieder ermöglichen technische Optionen Neues zu lernen. Was bedeutet es Mensch zu sein? Wie können wir über den Menschen nachdenken?

So stellt sich aus pädagogischer Sicht etwa die Frage nach dem Verhältnis zwischen Individuum und Daten. In der öffentlichen Diskussion werden z. B. Jugendliche, die in virtuellen Räumen Spuren hinterlassen, Selfies produzieren und Profile editieren, schnell als Selbstdarsteller betitelt, die sich anders darstellen würden, als sie wirklich sind. Dieser Vorwurf unterstellt, dass wir schon wüssten, wer wir sind und diese Identität dann richtig oder falsch darstellen könnten. In dieser Konzeption wird das Verhältnis des Selbst zu den das Selbst betreffenden Daten banalisiert. Entsprechend dem Konzept diskursiver Identität (vgl. Hall und DuGay 1996) sind wir jedoch ständig im Prozess herauszufinden, wer wir sind. Dafür nutzen wir Interaktionen, in denen wir Hypothesen über uns selbst prüfen. Ebenso nutzen wir die Reflexion im von uns erzeugten Profil. Etwas in eine äußere Form zu bringen, erlaubt uns, uns selbst zu verstehen. So wie wir im Schreiben, in der Auseinandersetzung mit dem entstehenden Text und seiner Struktur, herausfinden, was wir denken und nicht nur bereits Gedachtes zum Ausdruck bringen, so bringen wir unser Selbst in der Interaktion mit dem eigenen Profil in sozialen Medien in Erfahrung, indem wir eine Hypothese über uns selbst vergegenständlichen. Die Gestaltung eines eigenen Profils auf einer Social-Media-Plattform ermöglicht Reflexion und Entwicklung des Selbst in sozialer Interaktion und im Diskurs mit der eigenhändig erstellten Repräsentation. In diesem diskursiven Zugang zur Identität als Konstruktion, die niemals abgeschlossen ist (vgl. ebd.), nutzen wir die digitale Repräsentation als parasozialen Diskurspartner, mit dem wir die eigene Betrachtung prüfen. Wir beschreiben uns und lassen die Beschreibung zeitlich und situationsübergreifend wieder auf uns wirken. Die Auseinandersetzung mit den uns betreffenden Daten erschöpft sich somit nicht in einer getreuen Abbildung unserer Selbst, sondern ist selbst zentraler Bestandteil unserer Identitätsgenese.

Identität besteht in dieser emergenten Realität nicht nur aus Körpern, Verhalten und Interaktionen, sondern auch aus Daten. Wer ich bin, wird nicht zuletzt bestimmt durch die Daten, die ich erzeuge und ihre Verfügbarkeit für andere. Daten sind integraler Bestandteil des Selbst. Die Kapazität des Menschen, performativ zu sein, Kultur zu erzeugen, d. h. Praktiken zu reproduzieren und neu zu produzieren, sich im Sinne Hörnings (2004) als reflexiver Mitspieler auseinanderzusetzen mit dem Selbst und laufenden Praktiken, spiegelt sich auch im Konzept des digitalen Moratoriums wider. Das Digitale bietet Räume zum Selbstausdruck als Selbstreflexion, die im Sinne psychosozialer Moratorien des Erprobens genutzt werden. Psychosoziale Moratorien stehen entsprechend entwicklungspsychologischer Ansätze zur Genese einer eigenen Identität in der Gesellschaft offen. Sie ermöglichen, sich auszuprobieren, Grenzen ausloten und ein Selbst- und Weltverständnis zu erarbeiten. Das bedeutet selektives Gewährenlassen seitens der Gesellschaft und provokative Verspieltheit der Jugend als Selbstbehauptung innerhalb der Generationenkonkurrenz

\footnotetext{
${ }^{3}$ Ein Mitschnitt der Rede ist verfügbar unter https://www.youtube.com/watch?v=rUqZHSK7Rt4. Zugegriffen: 18. Dezember 2016.
} 
(vgl. Erikson 1973). In einer von Innovation und Dynamik geprägten Gesellschaft hat dieses Moratorium des Erprobens und anders Handelns eine gesamtgesellschaftliche Funktion (vgl. Oerter und Montada 1994). Böhnisch (vgl. 2008) hält fest: Das immer weiter eingeschränkte Moratorium wird durch ein vielfältiges unbeschränktes digitales Moratorium erweitert. Das sind digital konstituierte Räume, die Jugendliche ausgestalten, um mit Handeln und Andershandeln Praktiken zu erzeugen und sich selbst zu aktualisieren. In einer innovationsorientierten Gesellschaft hat Andershandeln und die produktive Kraft der Alterität eine große Bedeutung. Die über ein Individuum verfügbaren Daten sind in diesem Sinne lediglich als Sediment zurückliegender Versuche der Selbstaktualisierung zu lesen, ohne dass sie in einem zwingenden Zusammenhang zu zukünftigen Handlungsweisen und sich bildenden Identitäten stehen.

Barnett (2004) weist in diesem Zusammenhang darauf hin, dass wir uns in einer Zeit der Superkomplexität befinden, in der wir nicht nur in komplexe Systeme eingebunden sind, sondern es zudem auch immer eine Vielzahl nur bedingt kommensurabler Interpretationen dieser Systeme gibt. Infolgedessen wächst mit jeder neuen Erkenntnis auch unser Unwissen, so dass Unsicherheit und Unwissen zu zentralen Bestimmungsstücken des Handelns und Denkens werden. Dies schließt letztlich auch uns selbst und die Frage mit ein, wer wir sind. Der Umgang mit Unsicherheit, so Barnett, wandelt sich damit von einem vorrangig epistemischen zu einem ontologischen Problem für den Lerner. Es gibt keinen sicheren Bezugspunkt, um zu bestimmen, wer wir sind. Der Wandel und die damit verbundene Unsicherheit haben heute eine andere Qualität:

Now, what we are witnessing is a new kind of world order in which the changes are characteristically internal. They are primarily to do with how individuals understand themselves, with their sense of identity (or lack of it), with their being in the world; this is a world order which is characterized by ontological dispositions (Barnett 2004, S. 248).

Das bedeutet, dass unsere Beziehung zur Umgebung eine andere Funktion bekommen hat. Wir arbeiten nicht mit der Umgebung, sondern wir gehen mit der Umgebung, mit physischen und digital konstituierten Räumen um, um ein Verständnis unseres Selbst zu erarbeiten. Barnetts Konklusion ist dann: „I want to suggest, however, that the idea of skills, even generic skills, is a cul-de-sac. In contrast, the way forward lies in constructing and enacting a pedagogy for human being" (Barnett 2004, S. 247). Wie an späterer Stelle dieses Beitrags noch zu zeigen sein wird, betrifft die von Barnett beschriebene Unsicherheit nicht nur die Zukunft sondern auch gegenwärtige Situationen, insbesondere solche von pädagogischer Relevanz.

Die von Barnett beschriebene Unsicherheit in Folge parallel existierender und weitgehend unvereinbarer Interpretationsrahmen wird durch die Verbreitung datengetriebener Technologien noch weiter verstärkt. Aus praxistheoretischer Sicht werden diese Technologien selbst zum ,Produzent und Provokateur von Unbestimmtheiten“ (Hörning 2005, S. 308), indem sie sich in unsere Praktiken mischen, ohne dass wir in der Lage sind, sie vollständig zu entschlüsseln oder auch nur zu erkennen. Nur die aktive Auseinandersetzung mit diesen Technologien ermöglicht es uns, die den entsprechenden Praktiken innewohnenden Formen der Subjektivierung 
zu hinterfragen, ihren Geltungsanspruch zu unterlaufen und Anderes für möglich zu halten. So wie sich das Selbst durch die Aktualisierung und Neuproduktion von Praktiken entwickelt, konstituieren und verändern sich auch Individuum und Gesellschaft immer nur in Relation zu einander (vgl. Schatzki 1996, S. 13). Gesellschaft und Kultur sind keine abgeschlossenen, statischen Einheiten, sondern rücken als Prozesse der Vergesellschaftung in den Blick. Die praxeologische Perspektive betont die Offenheit, Instabilität und Veränderlichkeit von Individuum wie auch Gesellschaft (vgl. Schmidt 2012, S. 11). Unsere Praktiken verweben Technologie und Selbst. Verhalten, das Daten produziert, ist verwoben mit der Struktur und Materialität der Technologie. Oder in den Worten von Hörning (2005, S. 298):

Es ist der Umgang mit diesen Dingen, die Art und Weise, mit der sie behandelt, eingesetzt, verworfen, umgemodelt werden, die über ihr Schicksal in der Praxis entscheidet, eine Praxis, die wiederum durch den Eingang der technischen Dinge irritiert sowie provoziert wird und darüber zu neuen Reflexions- und Suchprozessen im Handeln und Verstehen Anlass gibt.

In diesem Sinne generieren digitale Dienste und Online-Plattformen keine Daten über uns, sie liefern keine Erkenntnis über menschliches Verhalten per se, sondern immer nur über unsere vorläufigen Versuche der Selbstaktualisierung in Auseinandersetzung mit den jeweiligen digitalen Angeboten, mit geschaffenen Strukturen bzw. normativen Affordanzen, die auf Annahmen und Konzepten ihrer Entwickler beruhen. Da Informationstechnologien Teil gesellschaftlicher Zusammenhänge sind, werden sie innerhalb gesellschaftlicher Praktiken konzipiert - die Konzepte, auf denen sie basieren, sind nicht neutral, sondern Teil des praktischen und nicht notwendigerweise explizierten Wissens der Akteure im Entwicklungsprozess. Woher stammen diese Konzepte? Und welche Konzepte sind das? Aktuelle Lerntechnologien, die Individualisierung und Personalisierung des Lernens versprechen, basieren nicht auf einem neutralen Konzept von Individualisierung, sondern auf einem Konzept wie es in Communities (der Entwicklerinnen und Entwickler) selbstreferentiell erzeugt wird. Gleiches gilt für die Sammlung, Integration und Verarbeitung großer Datenmengen, wie sie derzeit unter dem Schlagwort Big Data diskutiert wird. Auch wenn die Daten maschinell gesammelt, miteinander in Beziehung gesetzt und auf Muster durchsucht werden, so erfassen sie doch immer nur unsere vergangenen Versuche der produktiven Nutzung aber auch der Subversion eben jener Technologien. Wissen und Informationen geraten nicht zu uns, sondern Menschen schaffen kognitive Nischen, Informationsnischen, die sie selbst ausstatten (vgl. Bardone 2011), indem sie Netzwerke von sozialen Kontakten und Literatur aufbauen. Das Entscheidende ist nicht nur das Vorhandensein implizit oder explizit gefasster Konzepte, sondern auch wie ein Konzept verstanden und gefasst wird. Die produzierten Daten sind nicht theoriefrei, da sie aus der Nutzung von Technologien erzeugt werden, deren jeweilige Entwicklung auf spezifischen Konzepten basiert.

Algorithmische Technologien, insbesondere trainierbare oder evolutionäre Algorithmen, können wir in ihrer Funktionsweise und der Art und Weise, wie sie sich in unsere Tätigkeiten mischen, nicht mehr vollständig erkennen. SmartSketching ${ }^{4}$

\footnotetext{
4 http://idea-garden.org/ideagarden-3/smart-sketching/. Zugegriffen: 28. April 2016.
} 
ist ein trainiertes System mit einem Clustering-Algorithmus. Seine Funktion ist es, in Skizzen Gruppen von ähnlichen Elementen zu erkennen und zu markieren. Das System ,kann“ dies, da ihm etliche Zeichnungen von Menschen zum Lernen zur Verfügung gestellt wurden. Darin haben menschliche Akteure Segmente bzw. Gruppen von Elementen markiert, die sie als zusammengehörig erachtet haben. Wenn man SmartSketching nutzt, soll der trainierte Algorithmus im Hintergrund passende Cluster finden und bei Bedarf anzeigen, so dass der Nutzer diese Elemente als Gruppe editieren kann (verschieben, löschen etc.). Was der Algorithmus genau gelernt hat und welche Unterscheidungen er trifft, ist nicht vorhersag- oder nachvollziehbar. Auch die Entwickler selbst können dies nicht. In der Interaktion mit dem System befinden wir uns in einer inhärent unbestimmten Situation.

Neben dieser technisch induzierten Unsicherheit wissen wir bei der Nutzung von Plattformen algorithmisch getriebener Unternehmen zudem oftmals auch nicht, ob etwa die spezifische Auswahl und Anzeige von Inhalten auf einem generischen Algorithmus oder einer im weitesten Sinne politischen Entscheidung der Manager basiert. In welcher Situation befinden wir uns also? Wir wissen es nicht. Situationen, in denen wir agieren, sind unbestimmt, ambivalent, handlungs- und deutungsoffen. Unser Wissen reicht nicht aus, sie zu verstehen. Deutungsschemata sind nicht gegeben und gehen den Situationen nicht voraus. Wir können Verständnis nur in gestaltender Auseinandersetzung mit der Situation erzeugen. Auch sind die Situationen zu komplex, um sie vollständig zu analysieren. Kreativ sind wir nicht, weil wir eine neue Situation herbeiführen wollen, sondern weil wir mit Situationen, die wir nicht verstehen, für die wir keine Routinen und Regeln besitzen, nur kreativ umgehen können. Kreative Praktiken, bzw. Kreativität ist in diesem Sinne eine Form der Interaktion mit der Welt, in der Individuen und/oder Gruppen versuchen sich produktiv mit einer andernfalls unbestimmten Situation auseinanderzusetzen und neue Ideen hervorzubringen (vgl. Richter und Allert 2016).

Wenn wir mit lernenden algorithmischen Systemen wie Facebook interagieren, wissen wir das Instrument, mit dem wir umgehen, nicht einzuschätzen. Algorithmen wirken in unsere Aktivität. Wir können uns weder über den Algorithmus vollständig informieren noch seinen Einfluss auf unsere Tätigkeit des Informierens abschätzen. Wir müssen aktiv werden, um zu verstehen. An etwas herumbasteln, erfinden, erschaffen, vortäuschen und intervenieren sind Formen des Verstehens. Den FacebookAlgorithmus kreativ auszutricksen, seinen vermeintlichen Zweck zu unterlaufen und seine Reaktion zu beobachten, wird zur Strategie, seine Funktionsweise zu Gesicht zu bekommen. ${ }^{5}$ Wir nutzen (de-)konstruktive Aktionen als Sonde in die Situation, als Instrument der Wissensgenerierung, Erkenntnis und Deutung. Das Spiel mit der Technologie ist ontogenetisch. Wir wollen Wirklichkeit erfahren und die Artefakte, mit denen wir in unseren sozialen Praktiken verwickelt sind, in Erfahrung bringen, zumal wir in diesen Praktiken, in denen Technologien konstitutiv verwoben sind, Subjekthaftigkeit erzeugen und praktisches Wissen erlangen.

Im Folgenden wird uns die Tatsache der inhärent unbestimmten Situation, in der wir uns in Interaktion mit Technologien befinden können, interessieren. Während Facebook zum Zweck von Studien mit der Timeline der NutzerInnen experimentiert,

\footnotetext{
5 Siehe Garling (2014).
} 
um deren Verhalten zu verstehen ${ }^{6}$, kann es den individuell Nutzenden nicht vollständig gelingen, durch Experimentieren und Herumspielen am System den Algorithmus der Timeline zu erkennen. Dadurch entsteht ein Machtgefälle. Wissen und Kompetenz ermöglichen das Erkennen der Mechanismen durch das Individuum nicht. Man könnte argumentieren, dass es magisch ist, wenn wir nicht wissen, wie es passiert, ebenso magisch, wie Menschen in früheren Zeiten Naturgewalten erlebt haben und diese weder erklären noch vorhersagen bzw. ihre Erwartungen daran ausrichten konnten. Allerdings sind Algorithmen und Natur in mindestens zwei Aspekten unterschieden: (1) Naturgesetze sind weitgehend statisch. Algorithmen jedoch sind veränderbar, auch ohne dass diese Veränderung transparent gemacht werden muss. Veränderungen werden ggf. in der Interaktion, wenn die Reaktion des Systems nicht den Erwartungen entspricht, vermutbar. (2) Algorithmen sind intentional gemacht. Im Gegensatz zu teleonomen Naturgesetzen sind sie teleologisch.

Auch das Machtverhältnis, das entsteht, weil wir nicht wissen, welche Daten andere von uns haben, können Individuen mit ihrer Medienkompetenz nicht auflösen. Interessanterweise ist es Individualisierung, die diese Situation verstärkt. Im Abgleich mit anderen gelingt es uns manchmal, uns die Funktionsweise eines Algorithmus teilweise zu erschließen, z. B. beim Abgleichen der Suchergebnisse von Google mit Anderen bei gleichem Suchbegriff. Wir wissen nicht, welche Muster aus unserem (kollektiven) Verhalten aggregiert wurden und in unsere Tätigkeiten hineinspielen. Wir befinden uns nicht in einer Situation, die bestimmt ist und in der wir uns auf eine unbestimmte Zukunft vorbereiten, sondern wir befinden uns im Hier und Jetzt in einer unbestimmten Situation, weil wir die Technologien nicht vollständig erkennen können, die sich in unsere Interaktionen, Beziehungen und Tätigkeiten des Informierens und Lernens mischen. In Interaktionsprozessen bilden wir Erwartungen an das Verhalten aller Beteiligten, handeln aus, in welcher Situation wir uns befinden, nutzen Interaktionen, um uns über uns selbst klar zu werden und bilden kollektiv Muster in Form von Praktiken aus.

Zusammenfassend ergeben sich aus der hier skizzierten Sichtweise vier grundlegende Fragestellungen zur pädagogischen Einordnung datengetriebener Technologien:

1. Welche Konzepte des Anwenders bzw. Lerners liegen der entsprechenden Technologie zugrunde und welche Modi der Subjektivierung werden befördert?

2. Welche Machtdispositive werden durch den Einsatz der Technologie befördert und in welcher Weise verschieben sich die Machtverhältnisse zwischen den Akteuren?

3. Welche Rolle spielt der Anwender bzw. Lerner bei der Generierung der Daten?

4. Welche Praktiken der Adaption und Affirmation, aber auch der Subversion und Suspension eröffnen sich?

\footnotetext{
${ }^{6}$ In einer experimentellen, massive-scale „Contagious Study“ zum Beispiel. Facebook hat Nachrichten mit spezifischen Qualitäten gelöscht und in der Kontrollgruppe randomweise gelöscht (vgl. Kramer et al. 2013).
} 


\section{Prozesse der Subjektivierung und Learning Analytics}

Die Bedeutung der vorangestellten Überlegungen lässt sich sowohl an Fragen der Standardisierung, des Educational Data Mining, der Gestaltung von MOOCs und intelligenten tutoriellen Systemen, der Personalisierung oder anderen lerntechnologischen Entwicklungen diskutieren. Sie sollen im Folgenden jedoch anhand der aktuellen Diskussion um „Learning Analytics“ veranschaulicht werden. Im Mittelpunkt der Betrachtung steht dabei das Verhältnis von Subjekt und Technologie, von Daten und Performativität in der Interaktion mit Lerntechnologien bzw. im Lernen und in Bildungsprozessen.

Mit der zunehmenden Verbreitung von Lernmanagementsystemen und sozialen Medien hat sich in den zurückliegenden Jahren ein reges Interesse an computergestützten Verfahren und Technologien zur Erfassung, Sammlung, Analyse und Bereitstellung von Daten über Lernende, Lernprozesse und Lernkontexte entwickelt. Entsprechende Ansätze, die unter dem Überbegriff der „Learning Analytics“ zusammengefasst werden, zielen auf das Verständnis und die Optimierung der Lernprozesse und der entsprechenden Lernumgebungen ab (vgl. Long und Siemens 2011). Je nach Zielsetzung werden die Ergebnisse entsprechender Analysen den Lernenden selbst, Lehrenden, Organisationen oder politischen Entscheidungsträgern zur Verfügung gestellt. Neben der Reflexion durch den Anwender sollen entsprechende Verfahren und Technologien vor allem die Modellierung und Vorhersage zukünftigen Verhaltens ermöglichen (z. B. Greller und Drachsler 2012).

Während Vertreter entsprechender Ansätze vor allem Fragen des Datenschutzes, des Austauschs und der Offenlegung von Daten, des Missbrauchs von Ergebnissen sowie den Bedarf an Kompetenzen zur Interpretation und Nutzung der Analysen als kritisch erachten (z. B. Campbell et al. 2007; Greller und Drachsler 2012), ist auch grundlegendere Kritik geäußert worden. Diese Kritik betrifft insbesondere den immer wieder anzutreffenden Datenpositivismus. Daten sprächen hiernach für sich selbst und ihnen käme eine objektive Bedeutung zu (vgl. Buckingham Shum 2012) ${ }^{7}$ sowie die konservative Grundausrichtung entsprechender Verfahren, die auf der Annahme basieren, dass vergangenen oder gegenwärtigen Verhaltensmustern ein prognostischer und prädiktiver Wert zukommt (vgl. Richter und Allert 2014). Vergangenes Verhalten könne zur Grundlage werden, weitere Verhaltensoptionen bereit zu stellen. ${ }^{8}$ Diese Kritikpunkte sind jedoch insofern generisch, als dass sie im Hinblick auf die Nutzung von Big Data zur Vorhersage von Verhalten in sozio-technoökonomischen Systemen generell gelten (z. B. Boyd und Crawford 2011; Reichert 2016).

So wichtig und berechtigt die genannten Fragestellungen und Kritikpunkte auch sind, so wenig sagen sie jedoch gerade über die pädagogischen Implikationen entsprechender Technologien aus. Die Reflexion der pädagogischen Implikationen der

\footnotetext{
${ }^{7}$ Selbst scheinbar triviale Operationen wie etwa die Zuordnung von Logfiledaten zu einer bestimmten Person erweisen sich in der Praxis als äußerst problematisch, kann doch ein Anwender seine Zugriffsdaten weitergeben oder mit Lernpartnern an einem gemeinsamen Rechner arbeiten.

${ }^{8}$ Ein Beispiel hierfür ist die Auswertung von vorhandenen Lebensläufen, um Anwender bei der eigenen Karriereplanung zu unterstützen.
} 
Learning Analytics erscheint jedoch umso bedeutsamer, als die entsprechenden Technologien (und ihre inhärenten Annahmen) gewöhnlich als pädagogisch neutral verstanden werden (z. B. Greller und Drachsler 2012). Pädagogisch neutral wären sie, wenn ihr Einsatz nicht auf das pädagogische Handeln konstitutiv einwirkt, bzw. wenn spezifische Formen und Vorstellungen von Lernen nicht besser unterstützt und nahegelegt werden als andere. Demgegenüber steht die Überzeugung, dass Technologien normative Affordanzen besitzen, die implizite oder explizite Annahmen über den Gegenstand (z. B. Lernen) vergegenständlichen.

Selbst wenn eingeräumt wird, dass konkrete Ansätze in diesem Bereich nicht neutral sein können, sondern in ihnen immer auch spezifische pädagogische Positionen und Beurteilungssysteme zum Tragen kommen (z. B. Buckingham Shum 2012), so werden jedoch die technologie-immanenten Annahmen aus der pädagogischen Analyse ausgeklammert. Vor diesem Hintergrund ist es wichtig, die technologischen Prämissen entsprechender Ansätze selbst zu hinterfragen und im Hinblick auf ihre pädagogischen Implikationen zu reflektieren. Im Folgenden konkretisieren wir deshalb die im vorangegangenen Kapitel erarbeiteten Fragestellungen in Bezug auf Learning Analytics:

1. Welches Menschenbild liegt Learning Analytics zugrunde und welche Modi der Subjektivierung werden befördert? Hildebrandt (2006) hat darauf hingewiesen, dass ein Individuum durch entsprechende Systeme als ein ,korreliertes Datensubjekt“ definiert wird, das durch sein vorausgegangenes Verhalten und seine „objektiven“ Eigenschaften bestimmt ist, während die radikale Unvorhersehbarkeit zukünftigen Verhaltens ausgeklammert wird. Welche Möglichkeiten hat die/der Einzelne vor diesem Hintergrund, ihre/seine eigene Identität zu entwickeln, wenn sie/er letztlich ,nur noch als dechiffrierbare und transformierbare Figur seiner Brauchbarkeiten ins Blickfeld rückt" (Reichert 2016, S. 23). Welche Bedeutung kommt der kritischen Reflexion des eigenen Verhaltens zu, wenn diese als systemimmanente Notwendigkeit erachtet und durch vorab spezifizierte Parameter getriggert und eingefordert wird?

2. Ist die Messung von Eigenschaften von Individuen eine Vorausbedingung für Lernangebote oder bereits eine Intervention? Learning Analytics produziert Daten und stellt keine Erhebung dar. Der hier hinterfragte Aspekt geht jedoch darüber hinaus. In der Entwicklung von Lerntechnologien und Learning Analytics werden Eigenschaften von Lernenden meist als Vorbedingung oder Voraussetzung von Maßnahmen und Lernprozessen gedacht. Aber: „Du hast in Mathe eine 5!“ kann nicht nur als Eigenschaft einer eigenen Leistung, sondern auch als Aussage über sich selbst verstanden werden. Es macht eine Aussage über mich, bzw. einen messbaren Aspekt meines Selbst: „Eine Mathe 5! - Das bin ich.“ Harris (1998) geht auf einen in diesem Zusammenhang beachtenswerten Mechanismus ein: Schülerinnen und Schüler sind sensibel für Äußerungen in ihrem Umfeld, die ihnen erlauben, eine Zuschreibung zu einer Kategorie bzw. Gruppe zu treffen wie: „Ich gehöre zu den Matheunbegabten“. Diese Zuschreibung ist dann erst der Ausgangspunkt, das Verhalten herzustellen, das den Mitgliedern der Gruppe dieser Kategorie zugeschrieben wird, z. B. die Hausaufgaben nicht machen. Sie stellen mit ihrem Verhalten die Gruppe und die Zuordnung zu dieser Gruppe her 
(Harris 1998). Daten sind Ausgangspunkt von Tätigkeiten, in denen Eigenschaften hergestellt werden - nicht die bloße Messung von Eigenschaften. Daten sind somit ein Bestandteil der perfomativen Subjektivierung.

3. Welche Machtdispositive werden durch den Einsatz von Learning Analytics befördert? Es geht hier um die Frage nach der Transparenz und Nachvollziehbarkeit der Datenverarbeitung. Ungeachtet des Anspruchs auf eine evidenzbasierte Vorgehensweise sind entsprechende Verfahren aus Sicht der Anwender für gewöhnlich weder transparent noch nachvollziehbar. Vielmehr werden Datensammlung und -verarbeitung systematisch aus der Anwendersicht ausgeblendet (vgl. Reichert 2016), sei es, weil die zugrundeliegenden statistischen Analyseverfahren nur maschinell ausführbar sind oder um ein Unterlaufen des Systems durch die Anwender zu unterbinden. In jedem Fall verändern sich jedoch nicht nur die Informationslage, sondern auch die Machtstrukturen zwischen den beteiligten Akteuren, inklusive der an der softwaretechnischen Umsetzung beteiligten Programmierern. ${ }^{9}$

4. Welche Praktiken sowohl der Adaption und Affirmation, aber auch der Subversion und Suspension eröffnen sich? Um Daten aus Lernsystemen für Learning Analytics wie angezielt nutzen zu können, muss sich der Lernende an das vorgegebene System anpassen und somit Adaption und Affirmation zeigen. Gleichzeitig erfordert selbstbestimmtes Handeln jedoch gerade auch die kritische Auseinandersetzung des Einzelnen mit diesen Technologien. Die Bedeutung dieser Technologien (und damit verbunden auch die Entwicklung der eigenen Identität) erschließt sich dabei gerade nicht nur in der planmäßigen Nutzung, sondern im Herumspielen und Austricksen des Systems bis hin zur Verweigerung der Nutzung. Sowohl die Anpassung als auch der Widerstand gegenüber Lernzielen und Lernformen, die die ältere Generation für die jüngere bzw. der eine für den anderen vorsieht, ist dabei letztlich auch ein politischer Akt. Die Situation in der Nutzung der Lerntechnologie ist für Lernende inhärent unbestimmt. Sie haben weder Einblick in die Intentionen der Entwickler noch in die hinter der Technologie liegenden Algorithmen und müssen sich entweder anpassen oder mit dem System herumspielen.

Die hier angerissenen Fragestellungen sollten verdeutlichen, dass Learning Analytics auch unabhängig von den spezifischen pädagogischen Theorien, die sie zu unterstützen suchen, alles andere als neutral sind. Vielmehr sind sie selbst Teil komplexer soziokultureller Entwicklungspfade und haben Auswirkungen weit über ihre unmittelbare Verwendung hinaus. Insofern sind sie wie andere Technologien auch „,neither good nor bad; nor is it neutral“ (Kranzberg 1986, S. 545).

Grundlegend muss die Idee, Lernen zu vermessen, zunächst anschlussfähig sein, um in Technologien vergegenständlicht zu werden. Das Konzept „Learning Analytics" wird also gedacht und realisiert, weil es in gegenwärtigen kollektiven Praktiken anschlussfähig ist.

Die Diskussion über Learning Analytics betrifft zum einen Vorstellungen über den Menschen sowie seine Beziehung zur Technologie. Funktionieren würden solche Systeme, wenn sich das Verhalten aus dem bereitgestellten System ableiten ließe und der Ablauf der Lernschritte gesteuert werden kann oder soll. Das eigensinnige

\footnotetext{
${ }^{9}$ Hierzu auch: Röhle (2010). Er analysiert Google-Algorithmen unter foucaultscher Perspektive.
} 
Herumspielen mit einem System, um es zu erkennen, der produktive Umgang mit Unbestimmtheit und Unsicherheit, der kreative Akt zur Erkenntnis, das produktive Umnutzen und die Performativität im Umgang mit dem Lernmanagementsystem machen Daten ,unbrauchbar“, sind jedoch Zeichen von Selbstbestimmung und Kreativität. Sie stehen den Lernenden zu, um das System zu erkennen und selbstbestimmt zu handeln.

Im Design wird die kreative Aneignung von Artefakten wie z. B. Software mit Begriffen wie Non Intentional Design (Brandes und Erlhoff 2006), Appropriation und Completing Design in Use (Carroll 2004) bedacht. Porombka (2015) bezeichnet solche, die ,nicht richtig mitmachen“ und Strategien gegen Berechenbarkeit ausspielen, als Artisten der Gelegentlichkeit. Andersrum beschreibt Stolterman ein Entwurfsmuster mit dem Titel „Pattern for Unintended Use“ ${ }^{10}$ mit dem Ziel, Technologie so offen zu gestalten, dass sie zur kreativen, unvorhersehbaren und unplanbaren Nutzung einlädt. ${ }^{11}$ In Bezug auf interaktive webbasierte Systeme ist Kreativität nicht auf die individuelle Nutzung, sondern auf kollektive Praktiken bezogen. Gleichzeitig machen bereits bestehende Praktiken Systeme anschlussfähig oder auch nicht. Insofern ist die Art der Nutzung, der Affirmation oder Subversion, in laufende kollektive Praktiken einbezogen, und nicht allein eine individuelle Entscheidung. Insofern gehen sowohl Ansätze zur Steuerung und Kontrolle von Lern- und Bildungsprozessen als auch solche zur Förderung von Selbstbestimmung und Kreativität immer mit entsprechenden Prozessen der Vergesellschaftung einher und können nicht isoliert betrachtet werden.

Hörning (2004) sieht uns als reflexive Mitspieler mit der Kapazität der Gleichzeitigkeit von Anpassung und Autonomie ausgestattet. Der reflexive Mitspieler kann sich auf Praktiken einlassen und gleichzeitig Distanz einnehmen und Eigensinn aufbringen. Reckwitz traut uns als Individuen in den Praktiken selbst Unberechenbarkeit und Improvisation zu, woraus Praktiken transformiert werden. Reckwitz (2013) schreibt über das Werden und Vergehen der sozialen Formen und die Unkalkulierbarkeit der Individuen:

Aus der Perspektive einer allgemeinen poststrukturalistischen Ontologie des Sozialen kann man zu Recht davon ausgehen, dass soziale ebenso wie psychische und organische Strukturen ganz generell in einem ständigen Prozess des Entstehens und Verschwindens, des Neuknüpfens und Auflösens begriffen sind. Auch wenn man an den Individuen und ihrer Alltagspraktik ansetzt, kann man ganz allgemein voraussetzen, dass in ihrem Verhalten trotz aller Routine immer schon Unberechenbares und Improvisiertes steckt (S. 16).

Wenn wir uns für soziale Praktiken als Bezugspunkt entscheiden, geht es weder darum, den Einzelnen aus der Verantwortung zu entlassen, noch die Rahmenbedingungen kleinzureden. Vielmehr geht es uns darum, den Umgang miteinander als Erfahrungs- und gestaltbaren Spielraum an der Schnittstelle zwischen Individuum

\footnotetext{
10 http://www.publicsphereproject.org/content/design-unintended-use. Zugegriffen: 28. April 2016.

11 Auch für das Lernen gibt es Technologien, die nicht den Ablauf, die Informationseinheiten oder den Zugang verwalten und steuern, sondern kollaborative kreative Teamarbeit und ein forschendes offenes Vorgehen unterstützen. Insofern ist dieser Artikel keine Kritik an Technologien in Lernprozessen per se.
} 
und kollektiven Praktiken ins Blickfeld zu rücken, wobei anders handeln die Möglichkeit birgt, durch Gestaltung die laufenden Praktiken in Erfahrung zu bringen.

Zum anderen betreffen Technologien und Algorithmen die Beziehung zwischen repräsentativen Demokratien und Unternehmen. Im Gegensatz zu aufkommenden Forderungen nach einem wirtschaftsförderlichen Datenreichtum hat etwa Aral Balkan ein Ethical Design Manifesto ${ }^{12}$ formuliert, das den Schutz bürgerlicher Rechte und demokratischer Prinzipien in der Technologieentwicklung einfordert. Dezentralisierte, Zero-Knowledge-Anwendungen, die ein Minimum an Informationen an Dritte übermitteln, peer-to-peer, Ende-zu-Ende-verschlüsselte, freie, interoperable, zugängliche und nachhaltige Open-Source-Technologien lassen sich ebenso entwickeln und nutzen, wie wir auch Forderungen nach Transparenz an große Internetkonzerne stellen müssen.

\section{Quintessenz und Ausgangspunkt}

Artefakte sind ein konstitutives Element in unseren Praktiken, in denen wir Subjekthaftigkeit erzeugen, so dass eine pädagogische Perspektive, die die Nutzung und den Umgang mit Technologien in den Blick nimmt, essentiell zu den Grundfragen der Pädagogik beitragen kann. Sie ist zwingend und entscheidend. Zu den Aufgaben einer solchen (Medien-)Pädagogik gehören die kontinuierliche Analyse technologischer Entwicklung und ihrer Nutzung, die Entwicklung theoriebasierter Perspektiven hierauf sowie die Ausarbeitung pädagogischer Fragen und Implikationen. Zudem gilt es, auf der Seite pädagogischen Handelns Modelle zu entwickeln und zu evaluieren, die zur Teilhabe an Gestaltung und Untersuchung sozio-technischer Systeme befähigen und ermächtigen.

Bildung und Lernen mit Medien sind von Erziehung und Sozialisation nicht zu trennen, da die Technologieentwicklung innerhalb gesellschaftlicher Praktiken stattfindet und Technologien, Konzepte und Annahmen vergegenständlichen. Es gibt eine Reihe von Fragen, die im Rahmen dieses Artikels keinen Platz mehr finden oder noch nicht zu beantworten sind.

Als Fazit arbeiten wir Konsequenzen für die Pädagogik als Wissenschaft heraus, die gleichzeitig Ausgangspunkt für ein entsprechendes Forschungsprogramm darstellen: 1) Lernen und Bildung lassen sich nicht losgelöst von den sozialen und materiellen Bezügen verstehen, in die sie eingebettet sind und die sie (re-)produzieren. Eine transaktionale Pädagogik erfordert somit den Bezug auf (Medien-)Theorien, die die soziale und materielle Dimension menschlichen Seins und Handelns in Rechnung stellen. 2) Gegenwart und Zukunft sind inhärent unbestimmt. Wir verstehen unbestimmte Situationen, indem wir in ihnen kreativ und kritisch intervenieren und sie gestalten - eine transaktionale Pädagogik versteht Kreativität und produktives Handeln als Form des Verstehens.

Weshalb sollten wir versuchen Entwicklungen einzuordnen, während sie uns bereits überholen? Vielleicht weil wir sie erst in der Auseinandersetzung, die durch Akzeptanz und Ablehnung entsteht, verstehen. Technologien können epistemische

12 https://ind.ie/ethical-design/. Zugegriffen: 28. April 2016. 
Objekte sein, die in ihrem Einsatz Fragen eröffnen. Können wir nicht auch als Wissenschaftlerinnen und Wissenschaftler epistemische Objekte in die Welt setzen? Nicht nur Texte, die unsere Ideen repräsentieren, sondern Objekte, die partiell sind und Fragen entwickeln? Können wir einen gestalterischen Weg finden, Objekte zu generieren, die mehr Fragen in sich bergen als wir absichtlich in sie hineinstecken?

Open Access Dieser Artikel wird unter der Creative Commons Namensnennung 4.0 International Lizenz (http://creativecommons.org/licenses/by/4.0/deed.de) veröffentlicht, welche die Nutzung, Vervielfältigung, Bearbeitung, Verbreitung und Wiedergabe in jeglichem Medium und Format erlaubt, sofern Sie den/die ursprünglichen Autor(en) und die Quelle ordnungsgemäß nennen, einen Link zur Creative Commons Lizenz beifügen und angeben, ob Änderungen vorgenommen wurden.

\section{Literatur}

Alkemeyer, T., \& Buschmann, N. (2016). Praktiken der Subjektivierung - Subjektivierung als Praxis. In H. Schäfer (Hrsg.), Praxistheorie: Ein soziologisches Forschungsprogramm (S. 115-136). Bielefeld: transcript.

Allert, H., Richter, C., Albrecht, J., \& Ruhl, E. (2015). Grabbling with the Not-Yet-Known. In University of Gothenburg (Hrsg.), Exploring the Material Conditions of Learning: The Computer Supported Collaborative Learning (CSCL) Conference 2015 (Conference Proceedings Bd. 1, S. 7-11). Gothenburg: University of Gothenburg, Gothenburg.

Asimov, I. (1950). I, Robot. New York: Gnome Press.

Balkan, A. (2016). Wir sind alle Cyborgs, ZEIT Online. http://www.zeit.de/digital/mobil/2016-03/ digitalisierung-big-data-soziale-netzwerke-ueberwachung-umgang-digital-denken/seite- 2 . Zugegriffen: 11. März 2016.

Bardone, E. (2011). Seeking chances: from biased rationality to distributed cognition. Berlin: Springer.

Barnett, R. (2004). Learning for an unknown future. Higher Education Research \& Development, 23(3), 247-260.

Belliger, A., \& Krieger, D. J. (2006). Einführung in die Akteur-Netzwerk-Theorie. In A. Belliger \& D. J. Krieger (Hrsg.), ANThology. Ein einführendes Handbuch zur Akteur-Netzwerk-Theorie (S. 13-50). Bielefeld: transcript.

Böhnisch, L. (2008). Sozialpädagogik der Lebensalter: Eine Einführung. Weinheim: Beltz Juventa.

Boyd, D., \& Crawford, K. (2011). Six provocations for big data. A decade in internet time: symposium on the dynamics of the Internet and society. Oxford Internet institute, oxford UK. http://papers.ssrn.com/ sol3/papers.cfm?abstract_id=1926431 (Erstellt: 21. September 2011). Zugegriffen: 30. Juni 2016.

Brandes, U., \& Erlhoff, M. (2006). Non intentional design. Köln: daab.

Buckingham Shum, S. (2012). Learning Analytics - Policy Brief. Moscow: UNESCO Institute for Information Technologies in Education. http://iite.unesco.org/pics/publications/en/files/3214711.pdf. Zugegriffen: 30. Juni 2016.

Campbell, J.P., DeBlois, P. B., \& Oblinger, D. G. (2007). Academic analytics. EDUCAUSE review, 42(4), 40-57.

Carroll, J. (2004). Completing design in use: closing the appropriation cycle. In Turku School of Economics and Business Administration (Hrsg.), Proceedings of the twelfth European conference on information systems (S. 337-347). Turku: Turku School of Economics and Business Administration.

Döring, N. (2003). Sozialpsychologie des Internet: Die Bedeutung des Internet für Kommunikationsprozesse, Identitäten, soziale Beziehungen und Gruppen. Göttingen: Hogrefe.

Erikson, E.H. (1973). Identität und Lebenszyklus. Frankfurt a. M.: Suhrkamp.

Garling, C. (2014). Tricking Facebook's Algorithm, The Atlantic. https://www.theatlantic.com/technology/ archive/2014/08/tricking-facebooks-algorithm/375801/. Zugegriffen: 31. August 2017.

Greller, W., \& Drachsler, H. (2012). Translating learning into numbers: a generic framework for learning analytics. Educational Technology \& Society, 15(3), 42-57.

Hall, S., \& DuGay, P. (1996). Questions of cultural identity. London, Thousand Oaks: SAGE.

Harris, J. R. (1998). The nurture assumption: why children turn out the way they do. New York: Touchstone.

Hildebrandt, M. (2006). Privacy and identity. In E. Claes, A. Duff, \& S. Gutwirth (Hrsg.), Privacy and the criminal law (S. 61-104). Antwerp: Intersentia.

Hörning, K. H. (2001). Experten des Alltags. Weilerswist: Velbrück. 
Hörning, K.H. (2004). Soziale Praxis zwischen Beharrung und Neuschöpfung. Ein Erkenntnis- und Theorieproblem. In K. H. Hörning \& J. Reuter (Hrsg.), Doing Culture - Neue Positionen zum Verhältnis von Kultur und sozialer Praxis (S. 19-39). Bielefeld: transcript.

Hörning, K.H. (2005). Lob der Praxis. Praktisches Wissen im Spannungsfeld technischer und sozialer Uneindeutigkeiten. In G. Gramm \& A. Hetzel (Hrsg.), Unbestimmtheitssignaturen der Technik. Eine neue Deutung der technisierten Welt (S. 297-301). Bielefeld: transcript.

Jörissen, B. (2015). Bildung der Dinge: Design und Subjektivation. In B. Jörissen \& T. Meyer (Hrsg.), Subjekt Medium Bildung, Medienbildung und Gesellschaft (Bd. 28, S. 215-233). Wiesbaden: Springer.

Knorr Cetina, K. (2001). Objectual practice. In T. R. Schatzki, K. Knorr, Cetina \& E. von Savigny (Hrsg.), The practice turn in contemporary theory (S. 175-188). London: Routledge.

Knuuttila, T. (2005). Models as epistemic artefacts: toward a non-represenationalist account of scientific representation (PhD-thesis. University of Helsinki, Helsinki, Finland). http://www.helsinki. fi/cradle/documents/Doctoral\%20dissertations/Knuuttila\%202005\%20Models\%20as\%20Epistemic \%20Artefacts.pdf. Zugegriffen: 30. Juni 2016.

Kramer, A. D. I., Guilloryb, J.E., \& Hancock, J. T. (2013). Experimental evidence of massive-scale emotional contagion through social networks. In National Academy of Sciences of the United States of America (Hrsg.), Proceedings of the National Academy of Sciences of the United States of America (Bd. 111 Nr. 24, S. 8788-8790). Washington, DC: National Academy of Sciences of the United States of America.

Kranzberg, M. (1986). Technology and history: "Kranzberg's laws”. Technology and Culture, 27(3), 544-560.

de Lagasnerie, G. (2016). Die Kunst der Revolte - Snowden, Assange, Manning. Berlin: Suhrkamp.

Long, P., \& Siemens, G. (2011). Penetrating the fog - analytics in learning and education. EDUCAUSE review, 46(5), 31-40.

Nowotny, H., \& Testa, G. (2009). Die gläsernen Gene. Die Erfindung des Individuums im molekularen Zeitalter. Frankfurt a. M.: Suhrkamp.

Oerter, R., \& Montada, L. (1994). Entwicklungspsychologie. Weinheim: Beltz.

Orlikowski, W. J. (2007). Sociomaterial practices: exploring technology at work. Organization Studies, 28, $1435-1448$.

Porombka, S. (2015). Komm ich heut nicht, komm ich morgen. DIE ZEIT, Nr. 51. http://www.zeit.de/2015/ 51/abwechslung-gelegenheit-spontanitaet-lebenseinstellung/komplettansicht (Erstellt: 31. Dezember 2015). Zugegriffen: 28. April 2016.

Reckwitz, A. (2009). Praktiken der Reflexivität. In F. Böhle \& M. Weihrich (Hrsg.), Handeln unter Unsicherheit (S. 169-182). Wiesbaden: Springer VS.

Reckwitz, A. (2013). Die Erfindung der Kreativität: Zum Prozess gesellschaftlicher Ästhetisierung. Berlin: Suhrkamp.

Reichert, R. (2016). Das Politische der Großdatenforschung. Politikum, 2(1), 20-31.

Richter, C., \& Allert, H. (2014). Moves beyond critique: design as inquiry as a form of critical engagement. In University of Stirling (Hrsg.), Proceedings of professional practice, education and learning (proPEL) 2014 (S. 25-27). Stirling: University of Stirling.

Richter, C., \& Allert, H. (2016). A practice-oriented perspective on creative design. International Journal of Design Creativity and Innovation, 4, 195-205.

Röhle, T. (2010). Der Google-Komplex. Über Macht im Zeitalter des Internets. Bielefeld: transcript.

Schatzki, T. (1996). Social Practices. A Wittgensteinian approach to human activity and the social. Cambrigde: University Press.

Schmidt, R. (2012). Soziologie der Praktiken: Konzeptionelle Studien und empirische Analysen. Berlin: Suhrkamp. 